\title{
ROAD DAMAGE EXTRACTION FROM POST-EARTHQUAKE UAV IMAGES ASSISTED BY VECTOR DATA
}

\author{
Zihan Chen, Aixia Dou* \\ Institute of Earthquake Forecasting, China Earthquake Administration, Beijing 100036, China - (chenzihanb, axdothy)@163.com
}

\section{Commission III, ICWG III/IVa}

KEY WORDS: Road damage extraction, Damage assessment, Earthquake, UAV images, Object-oriented classification, Threshold detection

\begin{abstract}
:
Extraction of road damage information after earthquake has been regarded as urgent mission. To collect information about stricken areas, Unmanned Aerial Vehicle can be used to obtain images rapidly. This paper put forward a novel method to detect road damage and bring forward a coefficient to assess road accessibility. With the assistance of vector road data, image data of the Jiuzhaigou Ms7.0 Earthquake is tested. In the first, the image is clipped according to vector buffer. Then a large-scale segmentation is applied to remove irrelevant objects. Thirdly, statistics of road features are analysed, and damage information is extracted. Combining with the on-filed investigation, the extraction result is effective.
\end{abstract}

\section{INTRODUCTION}

Earthquake is one of the most devastating natural disasters with extremely high risk, bringing huge lose to human's life and property safety. The following secondary geologic disasters, such as landslide, collapse and debris flow, usually cause damage to roads network. The roads network, considered as transportation lifeline, has a critical impact on rescue and reconstruction missions after earthquake. Therefore, the importance of acquiring road conditions timely is taken-for-granted. Via road damage information, it's possible for decision makers to obtain the transport accessibility and arrange relief routes. Thus, the extraction and assessment of road damage information is quite necessary.

Due to recent advancements in remote sensing technology, various data sources are provided which covers abundant ground information and can be acquired from satellite, aircraft, or many other platforms. However, conventional road damage extractions and assessment techniques using experts' experience to interpret and recognise remote sensing images visually are timeconsuming and the accuracy sometimes can be easily affected by subjective feelings(Wang et al. 2015).

In that case, with the help of remote sensing imagery and fastgrowing algorithm, post-earthquake damage extraction and assessment work can be done quickly and effectively(Mitomi et al. 2002; Chiroiu \& André 2001). Many researches have concentrated on using satellite optical image or SAR data, to get the details of collapsed buildings after earthquakes(Matsuoka \& Yamazaki 2005; Yamazaki \& Matsuoka 2007; Voigt et al. 2011; Dong \& Shan 2013).

Since the fast development of Unmanned Aerial Vehicle(UAV), very high resolution(VHR) images can be obtained more easily. The location accuracy and image resolution have been improved. It provides massive amount of information for post-earthquake assessment, which greatly enrich the data source. It is possible to identify different kinds of earthquake damage. A lot of studies have been carried out to detect buildings and roads' destruction.
(Li, P., Xu, H., Song 2011; Haghighattalab et al. 2010; Li et al. 2008; Samadzadegan \& Zarrinpanjeh 2003; Rutzinger et al. 2006; Chaudhuri et al. 2012; Adams \& Huyck 2004; Gusella et al. 2004).

When it comes to road damage detection, because of road's shape and small-scale feature, it becomes a difficulty to improve the efficiency and correctness of algorithm and to remove irrelevant noise. A new method to extract road damage information and to assess road accessibility rapidly is needed. To solve these problem, with the assistance of vector road data, this paper applies a novel method to extract road damage information from UAV images and analysis the functionality of roads in stricken areas.

\section{METHOD}

\subsection{General Overview}

In this paper, the purpose is to extract road damage information after earthquake using UAV images and vector data and, on that basis, classify and assess road accessibility. The flowchart of this method is shown in Figure 1.

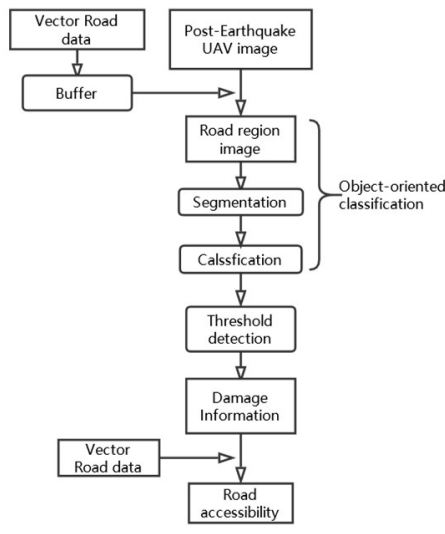

Figure 1. Flowchart of this method

* Corresponding author 
This method can be divided into four steps: image subset, objectoriented road regions extraction, damage extraction, and damage assessment. The first step can shorten necessary time for the rest parts and reduce redundant information which may result in enormous computation and H-resolution problem(Yu et al. 2006). Then, we get potential road region, and object-oriented classification is applied. Irrelevant disturbing details such as trees, shades, or cars are eliminated. After that, a second-time segmentation is carried out and according to the statistic results, threshold can be set. Threshold detection is then used to extract damage part of the roads. At last, by inspection the area and width of the broken parts, road accessibility can be determined, which moves further on the its application.

\subsection{Buffer and subset}

The first step is to identify possible road regions by using vector road data buffering to subset UAV images. Usually, in one UAV image, road networks are only a small part of the whole data. This is to reduce the calculation amount and speed up image processing. Roads have linear shape but different directions. Their width is sometimes stable owing to their class and usage. The width of buffer is set based on road grade.

In fact, there is a standard for road width: the expressway and first-class road usually has four lanes each with width of 3 meters. Main road of cities varies from 3 meters to 15-20 meters. Some second-class roads, or roads in plains, the lane's width comes to 7 meters, and roads of mixed traffic flow, the width is about 9-12 meters.

Given the width of road, the road vector is overlaid on UAV image. We use ArcGIS to create the buffer with specific width which exactly contains the whole road region and has the least unwanted information. Then subset the image.

The vector road we use in this paper has high accuracy both in location and shape. The next movement of our study is to extract and correct road vector which is not precise enough from images with different resolutions.

\subsection{Object-Oriented Road Regions Extraction}

Once road regions are extracted, objected-oriented classification is to be applied. To use object-oriented image classification, segmentation is needed. "Segmentation is the process of completely partitioning a scene (e.g., a remote sensing image) into non-overlapping regions (segments) in scene space (e.g., image space)."(Guo et al. 2004). the software "eCognition" can easily execute different kinds of segmentation algorithm. At most time, multiresolution segmentation is used to create image objects.

The multiresolution segmentation is the process to generate polygon objects with greatest homogeneity and least heterogeneity, it combines spectral feature and shape feature, and use this principle to merge pixels. With the higher resolution, the details of surface features are more abundant, and the heterogeneity of the image is greater(Wang et al. 2015).

By setting proper scale parameter and composition homogeneity criterion, which consists of two factor: shape and compactness, we take a group of pixels as representation the characteristics of ground targets rather than single pixels, so we treat each of the objects as a minimum classification unit for the following steps(Yu et al. 2006)
After segmentation, by using textures and brightness of object as constraint, we distinguish roads and non-roads objects. Through this process, classification result is achieved.

In this part, a large-scale parameter is to be set because we don't need to classify road region too specifically. The purpose is to remove violating objects, which is almost vegetation. And during this process, we assume that only two categories exist in the image: road (including both damaged and undamaged part) and vegetation. Once the violating objects are removed, we can infer the remaining ones are totally roads.

\subsection{Damage detection}

After roads objects are obtained, a grey-scale threshold method is proposed to detect damaged parts and extract their attribute. The detection process can be implied through four sections.

2.4.1 Segmentation: Owing to the input image data only contains road class, the segmentation needs to be fine enough to capture the anomaly of image, and the image patch should be small enough to represent the corresponding items. Pixels in the small patch normally have similar shape or spectral characteristic. In this step, the scale parameter is often much smaller than the previous part, in line with the space resolution of UAV images.

2.4.2 Statistics and threshold Set: Through last step, subobjects of roads are split. Descriptors should be chosen to reflect and translate the information hidden in the image. Common descriptors for damage objects determination, reveal spectral and textural information. We selected four features: Brightness (mean), GLCM Contrast, GLCM Standard Deviation and MaxDiff to describe and distinguish different objects. GLCM measures the local variations(Haghighattalab et al. 2010). By analysing these values, we can decide what threshold value should be. It is a method to detect road damage, object by object. We calculate the mean value of the whole road region, and draw a boxplot for each feature, the numbers fall out of the box is considered as outlying values, and the matching objects are considered damage information. We get a parameter $\mathrm{G}$ meaning the bounds of the box, then in each small, objects whose value is near to G (satisfy the formula 1) are counted and are regarded as undamaged road object.

$$
t_{0} \cdot \mathrm{G}_{1}<N<t_{1} \cdot \mathrm{G}_{2}
$$

$$
\begin{array}{ll}
\text { where } & t_{0}=\text { error factor } \\
t_{1}=\text { error factor } \\
\mathrm{G}_{1}=\text { lower bounds of boxplot } \\
\mathrm{G}_{2}=\text { upper bound of boxplot } \\
\mathrm{N}=\text { object feature value }
\end{array}
$$

2.4.3 Damage Object Determination: Based on threshold, assign damage class to objects and do post-processing. Under the influence of very high resolution of UAV images, centre lines of the roads appear yellow and sometimes obscure the results. Fortunately, this doesn't show often and it's easy to wipe out the impact. After this process, the damage information is ready to be exported and used.

\subsection{Assessment of road accessibility}

Assessment of road performance have proven useful in evaluating the seismic performance of lifeline infrastructure system(Chang \& Nojima 2001). Road accessibility can be categorized into three categories: clear, partially passable and totally blocked. Clear road means its functionality is in good 
condition, while "totally blocked" refers to a car cannot pass the damaged spot. When it comes to partially passable, we propose a coefficient $\rho$ to assess the damage degree of road and set correspondent threshold to define whether road is completely damaged or partially blocked (formula 2). After mentioned calculation, we can get basic information of road accessibility.

$$
\rho=\frac{W_{\text {damage }}}{W_{\text {road }}}
$$

where $\quad W_{\text {damage }}=$ width of damaged object

$\mathrm{W}_{\text {road }}=$ width of road

$\rho=$ road damage coefficient

Specifically, in accordance with road's level, county road is with the width of $4-8 \mathrm{~m}$. To ensure the minimum accessibility, $30 \%$ of its surface should be intact, which is $1.2-2.4 \mathrm{~m}$, similar to the width of small-sized vehicles. Therefore, if $\rho$ is a value greater than 0.7 , we assume that both lanes of the road are broken, and even small-sized vehicle cannot pass. When $\rho$ is between $0.2 \sim 0.7$, the road is regarded as partially passable.

\section{EXPERIMENTS AND RESULTS}

The study area, Jiuzhaigou is located at $100^{\circ} 30^{\prime}-104^{\circ} 27^{\prime} \mathrm{E}$, $30^{\circ} 35^{\prime}-34^{\circ} 19^{\prime} \mathrm{N}$, in the north-east part of Sichuan, China. An 7.0Ms earthquake occurred on $8^{\text {th }}$ August, 2017. After this earthquake, the main county road Z120 going through the scenic area of Jiuzhaigou is devastated, which brings huge loss to local people and great destruction to infrastructure.

We take UAV images obtained after the Jiuzhaigou Ms 7.0 earthquake as experimental data. The data is used to test the validity of this method. The image with three multispectral wavebands, was acquire on $12^{\text {th }}$ August 2017. It covers most of the heaviest stricken area, with an area of $8.77 \mathrm{~km}^{2}$. The space resolution of the imagery is $0.15 \mathrm{~m}$. And the overlaying road vector is prior data which fits the image accurately (shown in Figure 2).

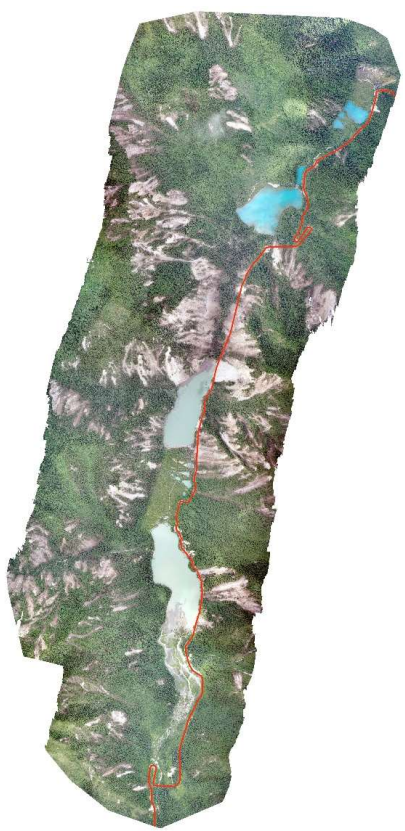

Figure 2. UAV images and road vector

To get buffer and subset image, we try two kinds of buffer radius: $5 \mathrm{~m}$ and $10 \mathrm{~m}$. Compared with each other, a best parameter can be decided. As is shown in Figure 3, image clipped by 5 m-radiusbuffer meets the requirement of contain whole information and is less effected by the covering vegetation. The file size was reduce by more than $50 \%$ to accelerate processing.

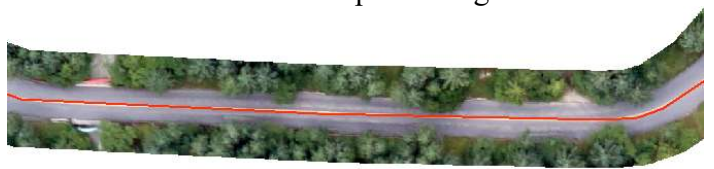

Figure 3(a). UAV images clipped by $10 \mathrm{~m}$ buffer

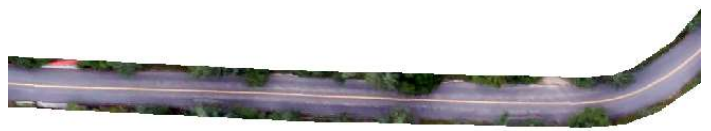

Figure 3(b). UAV images clipped by $5 \mathrm{~m}$ buffer

Then eCognition is used to apply multiresolution segmentation. For the purpose of removing vegetation, we set a scale parameter of 60 , shape factor for 0.1 , and 0.5 for compactness factor. The segmentation result is shown in Figure 4. As is mentioned in the method chapter, the large-scale segmentation makes it easy for us to identify vegetation.

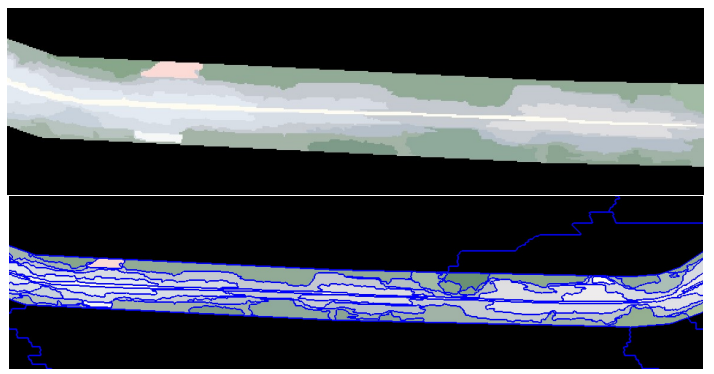

Figure 4. Large-scale Segmentation result (part)

Usually vegetation has apparent spectral and texture feature, thus, brightness and GLCM Contrast value is used to assign road class. By comparison of object features, we assign vegetation class under the condition that GLCM Contrast $\geqslant 390,0<$ Brightness $<180$. The rest objects with brightness $>0$ is classified into Road Region.(Figure 5)

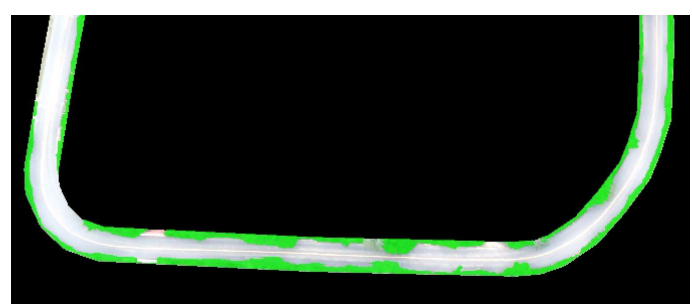

Figure 5. Vegetation class

The next step is aimed at the rest Road Region. Another multiresolution segmentation is carried out on a smaller scale. At the same time, weight of compactness factor is increased. The scale parameter is 10 with homogeneity criterion of 0.1 for shape and 0.7 for compactness.

After second-time segmentation, we export road region objects along with these features: Brightness, mean value of each band, GLCM Contrast, GLCM Standard Deviation (GLCM StdDev) and MaxDiff. 193 objects are achieved, and scatter plots for some features are drew (Figure 6). It is clearly seen in the figure that Brightness has a certain range representing undamaged road and the anomalous values indicate the ruins. 


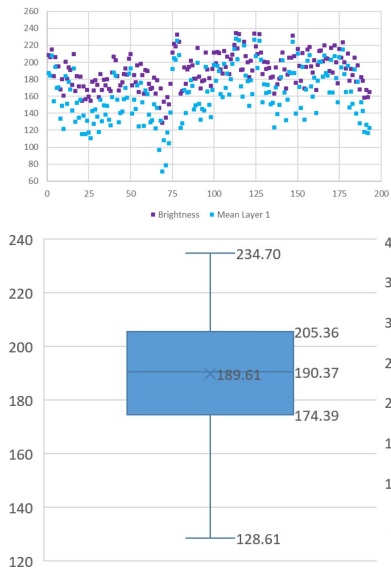

Brightness
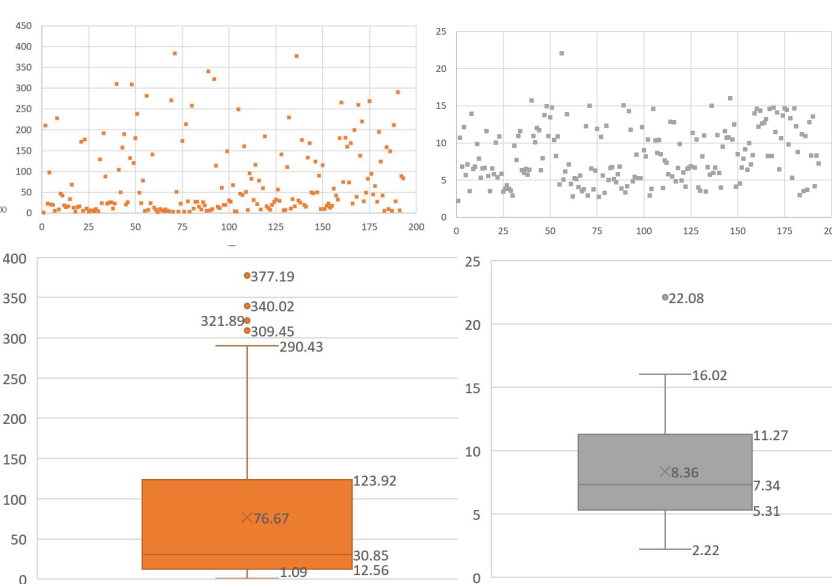

GLCM Contrast

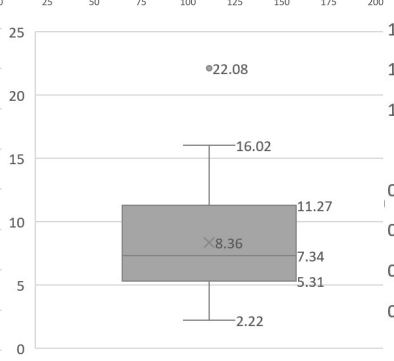

GLCM StdDev

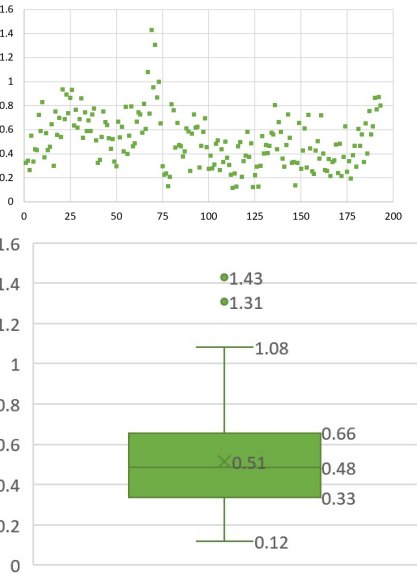

MaxDiff

Figure 6. Scatter plots and Boxplots of Road objects

Bounds of the boxplot shown in the picture above, the value of $\mathrm{G}_{1}$ is $\mathrm{Q}_{1}-1.5 \mathrm{IQR}$, and $\mathrm{G}_{2}$ value equals to the upper-quartile $\mathrm{Q}_{3}$ for formula 1. In this case, the threshold we use is that $G_{1}=128$, $G_{2}=205$. As for error factors, we set $t_{0}=1.07$ and $t_{1}=0.97$. Therefore, we consider objects with brightness greater than 200, or smaller than 120 as road damage class. As a result, the whole UAV images are divided into three categories: Vegetation, road, and road damage (Figure 7). Exporting the polygon and point feature layer, damage information extraction result is clearly displayed in Figure 8.

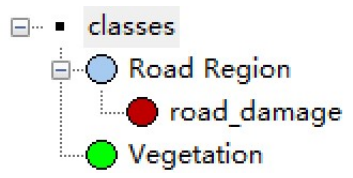

Figure 7. Class Hierarchy

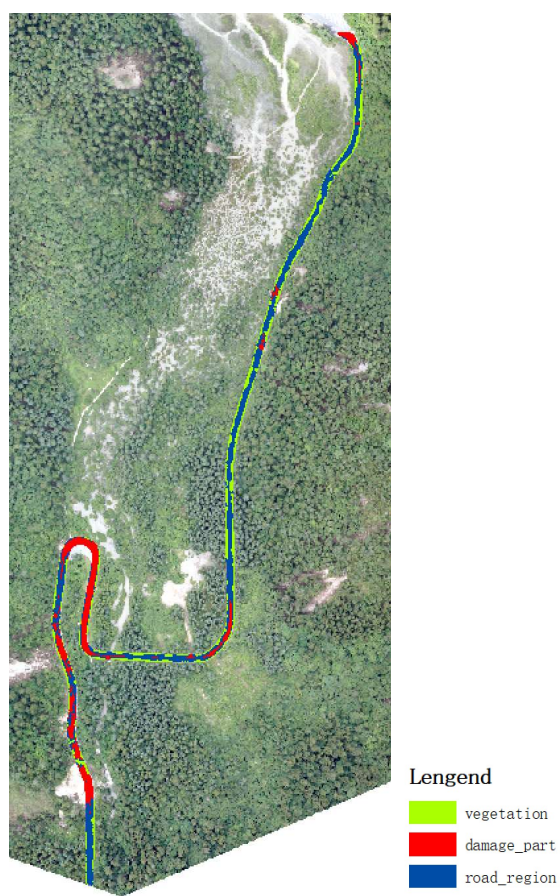

Figure 8. Exported Vector Layer
We detect 62 objects of damage in this area. The total area is $34.83 \mathrm{~m}^{2}$ (Figure 9). Comparing to on-filed investigation, 50 of them are correct, while the 12 wrong-classified object is because of some extreme high value part of images due to the imaging condition and angles of light. Besides, there is no damaged parts that are not detected in our research area, the extraction accuracy is nearly up to $81 \%$. Roads that are buried by landslide and rolling stones on the road can easily be detected with this method, however we have to admit that the vegetation dislocation causing road blockage is not easy to be picked up.
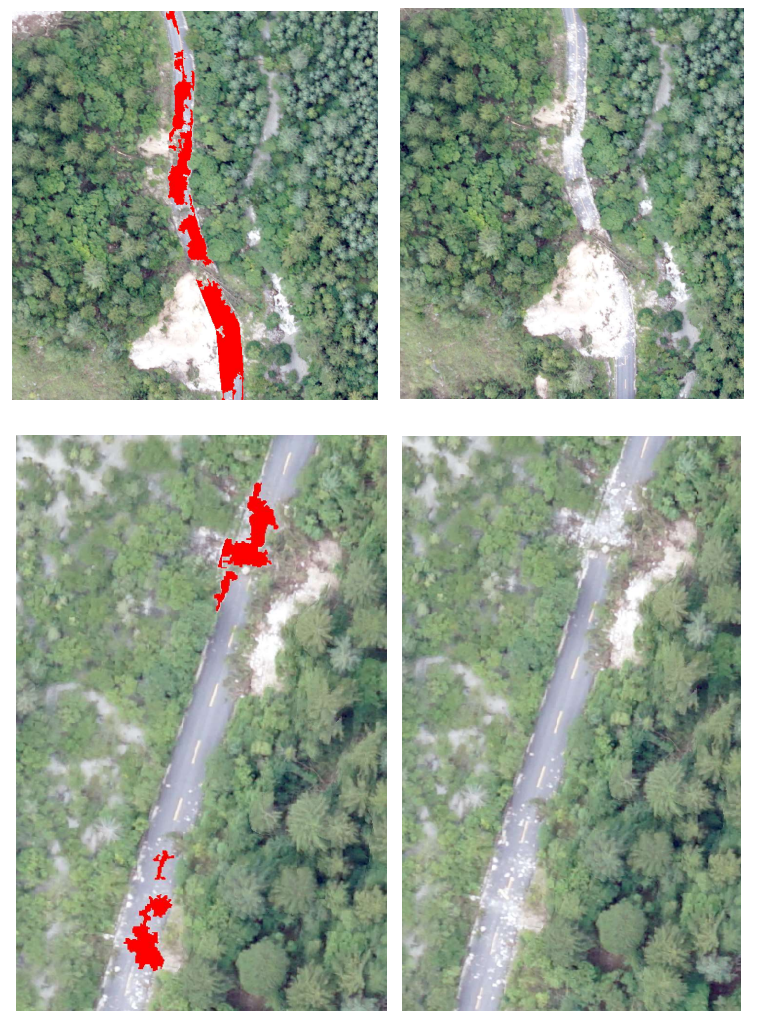

Figure 9. Damaged Road Examples

Take a closer look for damage assessment and road accessibility, we can get the attribute of restraining mass. Given the formula 2, width of obstacles is extracted, $\rho$ can be deduced to guide traffic route arrangement. Figure 10. shows three groups of road damage. 
In Figure 10(a) and (c), the dropping rubble stem the road, and $\rho$ value is greater than 0.7 , we infer that cars cannot pass these areas. In Figure 10(b) $\rho$ equals 0.44 , that means only half of the road surface is affected, small vehicles or experienced drivers can make it through. The most basic traffic capacity can still be provided.
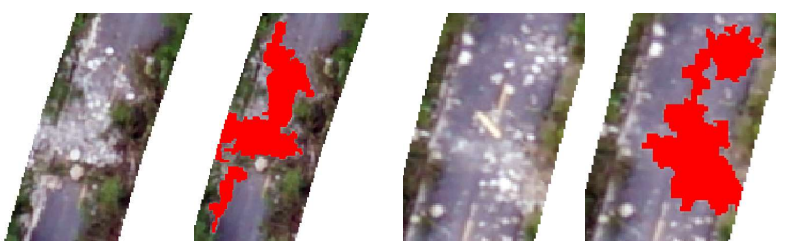

(a) $\rho=0.84$

(b) $\rho=0.44$

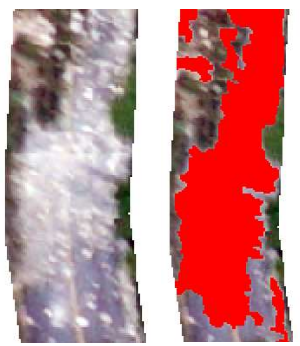

(c) $\rho=0.95$

Figure 10. Road Accessibility Assessment

\section{CONCLUSIONS}

In this paper, threshold detection method is proposed. A method for mono-temporal remote sensing image to extract damage information is applied. With the assistance of vector road data, images and road region can be easily located and the total data size is greatly reduced. Meanwhile, post-earthquake UAV images provide abundant details, which helps computer-aid damage detection and extraction become more accurate. The violating objects like vegetation and building can be dislodged by a larger-scale segmentation, and sub-slicing only for road region makes it possible to identify on damage spot a smaller scale. For brightness threshold detection, the objects statistics and threshold set can be designed as automatic process, at the same time, the result is convincing.

Admittedly, there remains some unsolved problems. Over bright undamaged road area will influence the final result and centre lines confuses the computer for wrongly take them as exceptional spectral value. What's more the efficiency and parallelization still look for further elevation.

\section{ACKNOWLEDGEMENTS}

This study is supported by National Key Research and Development program of China (2017YFB0504104).

\section{REFERENCES}

Adams, B., et al., 2004. Application of high-resolution optical satellite imagery for post-earthquake damage assessment: The 2003 boumerdes (algeria) and bam (iran) earthquakes. MCREER research and Accomplishments 2003-2004, pp.173186.

Chang, S.E. \& Nojima, N., 2001. Measuring post-disaster transportation system performance: The 1995 Kobe earthquake in comparative perspective. Transportation Research Part A: Policy and Practice, 35(6), pp.475-494.

Chaudhuri, D., Kushwaha, N.K. \& Samal, A., 2012. Semiautomated road detection from high resolution satellite images by directional morphological enhancement and segmentation techniques. IEEE Journal of Selected Topics in Applied Earth Observations and Remote Sensing, 5(5), pp.1538-1544.

Chiroiu, L. \& André, G., 2001. Damage Assessment Using High Resolution Satellite Imagery: Application To 2001 Bhuj, India, Earthquake. In Proc. 7th National Conference on Earthquake Engineering. pp. 1-13.

Dong, L. \& Shan, J., 2013. A comprehensive review of earthquake-induced building damage detection with remote sensing techniques. ISPRS Journal of Photogrammetry and Remote Sensing, 84, pp.85-99.

Guo, D., Weeks, A. \& Klee, H., 2004. Segmentations of road area in high resolution images. In Geoscience and Remote Sensing Symposium, 2004. IGARSS'04. Proceedings. 2004 IEEE International. pp. 3810-3813.

Gusella, L. et al., 2004. Damage Assessment with Very-High Resolution Optical Imagery Following the December 26, 2003 Bam, Iran Earthquake. , pp.1-6.

Haghighattalab, a. et al., 2010. Post-earthquake road damage assessment using region-based algorithms from high resolution satellite image. SPIE 7830, Image and Signal Processing for Remote Sensing XVI, pp. 78301E

Li, P., Xu, H., Song, B., 2011. A novel method for urban road damage detection using very high resolution satellite imagery and road map. Photogrammetric Engineering \& Remote Sensing, 77(10), pp.1057-1066.

Li, M. et al., 2008. Post-earthquake assessment of building damage degree using LiDAR data and imagery. Science in China, Series E: Technological Sciences, 51(suppl 2).pp.133143

Matsuoka, M. \& Yamazaki, F., 2005. Building damage detection using SAR intensity images for recent earthquakes. European Space Agency, (Special Publication) ESA SP, 0(572), pp.2021-2026.

Mitomi, H., Matsuoka, M. \& Yamazaki, F., 2002. Application of automated damage detection of buildings due to earthquakes by panchromatic television images. 7th U.S. National Conf. On Earthquake Engineering, CD-ROM

Rutzinger, M. et al., 2006. Object based analysis of airborne laser scanning data for natural hazard purposes using open source components. 1st International Conference on Objectbased Image Analysis, p.5.

Samadzadegan, F. \& Zarrinpanjeh, N., 2003. Earthquake Destruction Assessment of Urban Roads Network Using Satellite Imagery and Fuzzy Inference Systems. The International Archives of the Photogrammetry, Remote Sensing and Spatial Information Sciences, pp.409-414.

Voigt, S. et al., 2011. Rapid damage assessment and situation mapping: learning from the 2010 Haiti earthquake. 
Photogrammetric Engineering \& Remote Sensing, 77(9), pp.923-931.

Wang, J. et al., 2015. Road network extraction: a neuraldynamic framework based on deep learning and a finite state machine. International Journal of Remote Sensing, 36(12), pp.3144-3169.

YAMAZAKI, F. \& MATSUOKA, M., 2007. Remote Sensing Technologies in Post-Disaster Damage Assessment. Journal of Earthquake and Tsunami, 1(3), pp.193-210.

Yu, Q. et al., 2006. Objectbased detailed vegetation classification with airborne high spatial resolution remote sensing imagery. Photogrammetric Engineering and Remote Sensing, 72(7), pp.799-811. 